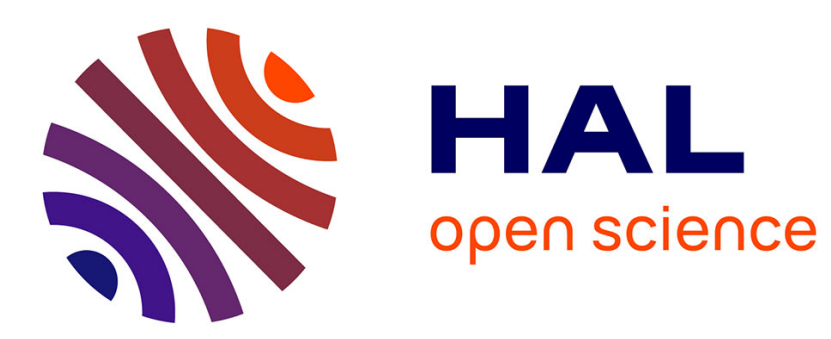

\title{
A cavity-by-cavity description of the aeroacoustic instability over a liner with a grazing flow
}

Xiwen Dai, Yves Aurégan

\section{To cite this version:}

Xiwen Dai, Yves Aurégan. A cavity-by-cavity description of the aeroacoustic instability over a liner with a grazing flow. Journal of Fluid Mechanics, 2018, 852, pp.126-145. 10.1017/jfm.2018.542 . hal-02337036

\section{HAL Id: hal-02337036 \\ https://hal.science/hal-02337036}

Submitted on 29 Oct 2019

HAL is a multi-disciplinary open access archive for the deposit and dissemination of scientific research documents, whether they are published or not. The documents may come from teaching and research institutions in France or abroad, or from public or private research centers.
L'archive ouverte pluridisciplinaire HAL, est destinée au dépôt et à la diffusion de documents scientifiques de niveau recherche, publiés ou non, émanant des établissements d'enseignement et de recherche français ou étrangers, des laboratoires publics ou privés. 


\title{
A cavity-by-cavity description of the aeroacoustic instability over a liner with a grazing flow
}

\author{
Xiwen Dai ${ }^{1} \dagger$ and Yves Aurégan ${ }^{2}$ \\ ${ }^{1}$ School of Mechanical Engineering, Shanghai Jiao Tong University, Shanghai 200240, China \\ ${ }^{2}$ Laboratoire d'Acoustique de l'Université du Maine, UMR CNRS 6613 Av. O Messiaen, \\ F-72085 LE MANS Cedex 9, France
}

(Received xx; revised $\mathrm{xx}$; accepted $\mathrm{xx}$ )

This paper presents a two-dimensional (2D) cavity-by-cavity description of a convective instability near a lined wall with low dissipation due to the coupling of hydrodynamic modes with resonance of the wall. For a liner consisting of an array of deep cavities periodically placed along a duct containing a mean shear flow, the acoustic and hydrodynamic disturbances are described by the linearized Euler equations. The Bloch modes and the scattering matrix of periodic cells are used to examine the instability over the liner. The unstable Bloch mode is due to the coupling of a hydrodynamic mode in the shear flow with the cavity resonance. It is demonstrated that even when all the transverse modes are stable in the duct-cavity system, i.e. when the Kelvin-Helmholtz instability of the shear flow over the cavities does not occur, such an instability over the liner can still exist. The unstable Bloch wave, excited by the incident sound wave at the upstream part of the liner, convectively grows along the liner, and regenerates sound near the downstream edge of the liner with a sound level higher than that incident sound level. It is shown that a homogenized approach, where the wall effect is described by a homogeneous impedance, can also explain the unstable behaviour above the liner. It reveals that a small wall resistance and a small and positive reactance are two necessary conditions for such an instability.

Key words:

\section{Introduction}

Liners are widely used to mitigate noise emissions from ducts. In most of the practical applications, from air conditioning systems to aero-engines, a flow is present in the duct. The interaction between flow and sound in the vicinity of a lined wall is complex and, therefore, a precise description of the flow-acoustic coupling near a liner still attracts a lot of attention (Tam et al. 2014; Zhang \& Bodony 2012, 2016; Khamis \& Brambley 2016, 2017). Surprisingly, under certain circumstances, instead of being attenuated, the sound can be amplified by a liner with a grazing flow. Indeed, it was observed that the transmission coefficient of a plane wave propagating through a liner in the flow direction can have a peak in amplitude larger than unity near the resonance frequency of the liner (Brandes \& Ronneberger 1995; Ronneberger \& Jüschke 2007; Aurégan \& Leroux

$\dagger$ Email address for correspondence: xiwen.dai@sjtu.edu.cn 
2008). Such a sound amplification appears at high flow velocities when the liner has a low resistance. The fluctuating fields, the convection velocity, and the growth rate of these unstable surface modes have been measured with optical technics (Marx et al. 2010). Those measurements have shown that the unstable disturbance near the lined wall increases exponentially along the liner with a relatively low amplification rate. Since no saturation mechanism appears experimentally, a linear approach to this problem seems possible. It can also be noted that such convectively unstable modes may also exist above porous materials (Aurégan \& Singh 2014; Alomar \& Aurégan 2017). In this case, the behaviour of unstable disturbances is more complex due to a slight flow inside the porous material which leads to separation on the downstream end of the material.

Many theoretical works have been performed on sound propagation in a lined duct with flow (Tester 1973; Koch \& Mohring 1983; Rienstra 2003; Brambley \& Peake 2006). A uniform mean flow was often assumed. To account for the effect of the infinitely thin vortex sheet on liner surface, the Ingard-Myers impedance boundary condition that requires the continuity of particle displacement across the vorticity sheet, is enforced (Ingard 1959; Myers 1980). With a uniform flow assumption plus the Ingard-Myers condition, a convective unstable mode (Tester 1973) and a surface unstable mode (Rienstra 2003) have been detected. However, some problems linked to the Ingard-Myers condition have been revealed: for instance, it is ill-posed in the time domain (Brambley 2009), and it can over-predict sound attenuation by over $10 \mathrm{~dB}$ compared to the results from the linearized Euler equations (Gabard 2013). To overcome the problem of ill-posedness, profiles with small but finite-thickness boundary layers were taken into account in impedance boundary conditions (Brambley 2011; Rienstra \& Darau 2011; Khamis \& Brambley 2016). These models are able to predict an absolutely or convectively unstable mode over the lined wall. In the modeling of the flow-acoustic coupling near the lined wall, the importance of taking into account viscosity was demonstrated (Aurégan et al. 2001; Marx \& Aurégan 2013; Khamis \& Brambley 2017). Including turbulent viscosity leads to more accurate predictions of the the growth rate and the velocity shape of the unstable surface mode above a liner. Also, the instability over the liner is influenced by the spatial development of the mean flow along the lined duct (Marx et al. 2010; Xin et al. 2016). All these efforts, however, are based on the assumption of a homogeneous impedance of the liner whereas in practice, this liner is constituted by a large number of small holes.

The limitations of the continuous impedance model in describing liners with flow have been revealed recently. It was first observed in experiments that two different impedances were measured according to whether the wave was propagating in the direction of flow or whether it was propagating against the flow (Renou \& Aurégan 2011). Since a uniform flow was assumed, the Ingard-Myers boundary condition was used in the impedance eduction from the experimental data. It could therefore be argued that the difference between these two impedances could be due to the imprecision of the impedance boundary condition. However, even taking into account the actual shear flow profile, the difference between the two impedances cannot be eliminated (Dai \& Aurégan 2016). The fact that liner impedance depends on flow direction has been verified in many laboratories, using different eduction methods and impedance boundary conditions (Spillere et al. 2017), and even using the linearized Navier-Stokes equations for wave propagation (Weng et al. 2018). From these experimental and theoretical works, we can draw a conclusion that the complex flow-acoustic coupling over the liner cannot be fully described by the currently used boundary conditions that involve a single quantity: an equivalent liner impedance.

The first objective of the present paper is, therefore, to examine the validity of the homogenized approach in describing the instability above a liner. To this end, a discrete approach of the aeroacoustic instability over a liner is developed. As sketched 

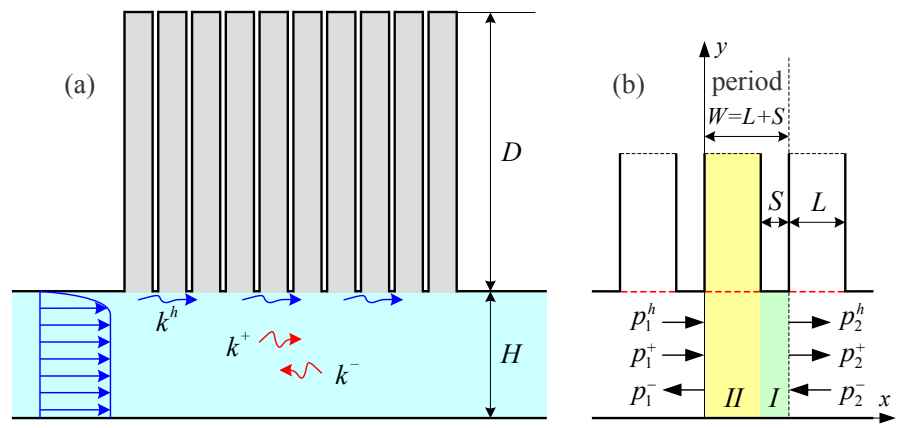

Figure 1. (Colour online) (a) Sketch of flow-acoustic coupling over a liner, (b) a periodic unit of the discrete model.

in figure 1, deep narrow cavities are periodically located along a duct containing a mean shear flow (period $W=L+S$ ). The spacing between the adjacent cavities $S$ is small compared to size $L$ of the cavity and very small compared to the acoustical wavelength. This liner can be seen as a two-dimensional (2D) version of the ones used in the previous experiments (Aurégan \& Leroux 2008; Marx et al. 2010). The acoustic and hydrodynamic disturbances are described by the linearized Euler equations (LEEs). To be able to describe the instability that appears in the experiments, some damping is required in the system. It is done artificially by adding a resistive layer at the inlet of the cavities and by adding an artificial damping to the hydrodynamic modes. This rough description of the viscous and turbulent effects only provides a qualitative comparison with the experiments, but it allows a better understanding of the mechanisms involved. For such periodic system, the wave propagation can be calculated using the Floquet-Bloch theorem, which states that the field can be split into a $2 \mathrm{D}$ periodic field modulated by a wave part of wavenumber $k_{B}$. The Bloch wave and the scattering matrix of the system can be used to study the instability over the liner due to flow-acoustic coupling. Section 2 explains the multimodal method used to compute the scattering in one-cell and multicell liners and the calculation of the Bloch waves is described. Using this model, the results of the periodic approach are presented and analyzed in section 3.1. It is first demonstrated that the aeroacoustic instability in the duct results from the coupling of a hydrodynamic mode in the boundary layer with the acoustic resonance of the cavities. Such an aeroacoustic instability can occur even if all transverse modes of the ductcavity system are stable. It is also demonstrated that for the unstable behaviour of the configuration shown in figure 1, a homogenized method based on an equivalent wall impedance is a reasonable approximation to the complete Bloch analysis. In section 3.2, a case where each periodic cell consists of two cavities of different depths is considered. It is found that a small depth difference can suppress the unstable behaviour above the liner, and explanations from both the Bloch and the homogenized point of view are given.

\section{Numerical model}

\subsection{Modal scattering in one cell}

In an inviscid perfect fluid when the nonlinear effects are neglected, the propagation of small disturbances about a steady mean flow can be described by the linearized Euler equations (LEEs): 


$$
\begin{aligned}
\left(\frac{\partial}{\partial t}+M_{0} f \frac{\partial}{\partial x}\right) u+M_{0} \frac{\mathrm{d} f}{\mathrm{~d} y} v & =-\frac{\partial p}{\partial x}, \\
\left(\frac{\partial}{\partial t}+M_{0} f \frac{\partial}{\partial x}\right) v & =-\frac{\partial p}{\partial y}, \\
\left(\frac{\partial}{\partial t}+M_{0} f \frac{\partial}{\partial x}\right) p & =-\left(\frac{\partial u}{\partial x}+\frac{\partial v}{\partial y}\right),
\end{aligned}
$$

where all the quantities have been put in dimensionless form using:

$$
p=\frac{p^{*}}{\rho_{0}^{*} c_{0}^{* 2}},(x, y)=\left(\frac{x^{*}}{H^{*}}, \frac{y^{*}}{H^{*}}\right),(u, v)=\left(\frac{u^{*}}{c_{0}^{*}}, \frac{v^{*}}{c_{0}^{*}}\right), \omega=\frac{\omega^{*} H^{*}}{c_{0}^{*}}, M_{0}=\frac{U_{0}^{*}}{c_{0}^{*}}, t=\frac{t^{*} c_{0}^{*}}{H^{*}},
$$

where $\rho_{0}^{*}$ is the mean density, $c_{0}^{*}$ is speed of sound, $U_{0}^{*}$ and $M_{0}$ are the average mean velocity and Mach number in the duct with the profile prescribed by the function $f(y)=M(y) / M_{0}, u^{*}$ and $v^{*}$ are the velocity disturbance in respectively the $x$ - and $y$-direction, $p^{*}$ is the pressure disturbance, $\omega^{*}$ is the angular frequency. In this paper, all the quantities with a star are quantities with dimensions while all the quantities without star are dimensionless quantities.

The variables are sought in the form:

$$
\begin{aligned}
& p=P(y) \exp (-\mathrm{i} k x) \exp (\mathrm{i} \omega t), \\
& v=V(y) \exp (-\mathrm{i} k x) \exp (\mathrm{i} \omega t),
\end{aligned}
$$

where $\mathrm{i}^{2}=-1$ and $k$ is dimensionless wavenumber. The equation (2.2) is written in term of these new variables as:

$$
\mathrm{i}\left(\omega-M_{0} f k\right) V=-\frac{\mathrm{d} P}{\mathrm{~d} y} .
$$

Removing the axial velocity from (2.1) and (2.3) leads to:

$$
\left(1-M_{0}^{2} f^{2}\right) k^{2} P+2 \omega M_{0} f k P-\omega^{2} P-\frac{\mathrm{d}^{2} P}{\mathrm{~d} y^{2}}=-2 \mathrm{i} M_{0} \frac{\mathrm{d} f}{\mathrm{~d} y} k V .
$$

This formulation of the equation is close to the classical Pridmore-Brown equation (Pridmore-Brown 1958) which is obtained when the value of $V$ extracted from $(2.5)$ is inserted in (2.6).

A unit cell of the duct-cavity system is split into 2 zones denoted by Roman number in figure 1(b). A shear flow is assumed in the duct, and its velocity profile is considered to be unchanged along the duct where $y<1$. The flow velocity inside the cavities, where $y>1$ is zero. To solve this problem of linear propagation in a shear flow, the multimodal method is used (Kooijman et al. 2008, 2010), where the disturbances in the ducts are expressed as a linear combination of acoustic modes and hydrodynamic modes. The equations (2.5) and (2.6) are discretized in the $y$-direction by taking $N_{1}$ equally spaced points in zone I, $N_{2}$ equally spaced points in zone II.

The spacing between interior points in all segments is $\Delta h=H / N_{1}=(H+D) / N_{2}$, and the first and last points are taken $\Delta h / 2$ from the the duct walls. Since the problem under study is known to lead to slope discontinuities in the profile of hydrodynamic modes, a low-order numerical scheme is used to solve the problem: the second-order centered finite difference method. The following generalized eigenvalue problem coming from (2.5) and(2.6) and using $Q=k P$ is formed in each of the segments: 


$$
k\left(\begin{array}{ccc}
\boldsymbol{I}-M_{0}^{2} \boldsymbol{f}^{2} & 2 \mathrm{i} M_{0} \boldsymbol{f}_{a} & \boldsymbol{0} \\
\mathbf{0} & \mathrm{i} M_{0} \boldsymbol{f} & \mathbf{0} \\
\mathbf{0} & \boldsymbol{0} & \boldsymbol{I}
\end{array}\right)\left(\begin{array}{l}
\boldsymbol{Q} \\
\boldsymbol{V} \\
\boldsymbol{P}
\end{array}\right)=\left(\begin{array}{ccc}
-2 \omega M_{0} \boldsymbol{f} & \boldsymbol{0} & \omega^{2} \boldsymbol{I}+\boldsymbol{D}_{2} \\
\boldsymbol{0} & \mathrm{i} \omega \boldsymbol{I} & \boldsymbol{D}_{1} \\
\boldsymbol{I} & \boldsymbol{0} & \boldsymbol{0}
\end{array}\right)\left(\begin{array}{l}
\boldsymbol{Q} \\
\boldsymbol{V} \\
\boldsymbol{P}
\end{array}\right),
$$

where $\boldsymbol{I}$ is the identity matrix, $\boldsymbol{f}, \boldsymbol{f}_{2}$ and $\boldsymbol{f}_{a}$ are diagonal matrices with on the diagonal the values of $f, f^{2}$ and $\mathrm{d} f / \mathrm{d} y$ at the discrete points in the ducts. $\boldsymbol{Q}, \boldsymbol{V}$, and $\boldsymbol{P}$ are the column vectors giving respectively the value of $Q(y), V(y)$ and $P(y)$ at the discrete points. $\boldsymbol{D}_{1}$ and $\boldsymbol{D}_{2}$ are matrices for the first and second order differential operators with respect to $y$. The boundary condition $\mathrm{d} p / \mathrm{d} y=0$ on the duct walls is taken into account in the differential operator matrices by introducing ghost points outside the duct walls. Solving the eigenvalue problem (2.7) (using the eig function of MATLAB) gives the eigenmodes and the corresponding wavenumbers in each zone. In the zone I, $3 N_{1}$ modes are found, including $N_{1}$ acoustic modes propagating or decaying (evanescent modes) in the $+x$ direction, $N_{1}$ acoustic modes propagating or decaying in the $-x$ direction, and $N_{1}$ hydrodynamic modes propagating in the $+x$ direction. In zone II, the mean flow velocity and its derivative are zero at discrete points where $y>1$. The last $N_{2}-N_{1}$ rows and columns of the middle parts in the matrices in (2.7) and the last $N_{2}-N_{1}$ elements of $V$ are skipped, corresponding to the no-flow part of this segment. Thus, there are $N_{2}$ acoustic modes propagating or decaying both in the $+x$ direction and in the $-x$ direction, and $N_{1}$ hydrodynamic modes propagating in the $+x$ direction in zone II.

To mimic the effects of a resistive sheet like a wiremesh or to mimic the cavity resistance due to thermo-viscous effects, a resistance $R$ denoted by the red dashed lines in figure $1(\mathrm{~b})$ is introduced at the mouth of each cavity. This resistance induces a pressure jump at $y=1$ for $0<x<L$ in zone II formulated as,

$$
\Delta p_{y=1}=R v_{y=1} \quad \text { for } \quad 0<x<L .
$$

The $n$th eigenvectors of (2.7) in the zone $j$ is ${ }^{t}\left(\boldsymbol{Q}_{n}^{j}, \boldsymbol{V}_{n}^{j}, \boldsymbol{P}_{n}^{j}\right)$, where $\boldsymbol{Q}_{n}^{j}, \boldsymbol{V}_{n}^{j}$, and $\boldsymbol{P}_{n}^{j}$ are the mode profiles of $q$ (Note $q=\mathrm{i} \partial p / \partial x), v$, and $p$, respectively. In each zone, the column vectors giving respectively the values of $Q(y), P(y)$, and $V(y)$ are written as a linear combination of the modes:

$$
\begin{aligned}
& \boldsymbol{Q}^{j}(x)=\sum_{n=1}^{N} C_{n}^{j} \boldsymbol{Q}_{n}^{j} \exp \left(-\mathrm{i} k_{n}^{j} x\right), \\
& \boldsymbol{P}^{j}(x)=\sum_{n=1}^{N} C_{n}^{j} \boldsymbol{P}_{n}^{j} \exp \left(-\mathrm{i} k_{n}^{j} x\right), \\
& \boldsymbol{V}^{j}(x)=\sum_{n=1}^{N} C_{n}^{j} \boldsymbol{V}_{n}^{j} \exp \left(-\mathrm{i} k_{n}^{j} x\right),
\end{aligned}
$$

where $C_{n}^{j}$ is the coefficient of the $n$th mode in zone $j$ and $N=3 N_{1}$ in zone I and $N=2 N_{2}+N_{1}$ zone II.

The modes in each duct segment are then matched using the continuity of pressure $p$, velocity $v$, and $\partial p / \partial x$ at the interfaces between zones I and II, and $\partial p / \partial x=0$ on the vertical walls inside the cavity. The continuity and wall conditions can be put in the form of a large matrix that links all the incoming waves in the cell to out-going waves and to all the internal variables. From this large matrix, the scattering matrix is written:

$$
\left(\begin{array}{l}
C_{2}^{+} \\
C_{1}^{-}
\end{array}\right)=\mathbf{S}\left(\begin{array}{l}
C_{1}^{+} \\
C_{2}^{-}
\end{array}\right)
$$


where vectors $C_{1}^{ \pm}$(resp. $C_{2}^{ \pm}$) contain the duct mode coefficients for $x=0$ (resp. $x=W$ ) for wave going in the flow direction (resp. for wave propagation opposite to the flow) and

$$
\mathbf{S}=\left(\begin{array}{cc}
\boldsymbol{T}^{+} & \boldsymbol{R}^{-} \\
\boldsymbol{R}^{+} & \boldsymbol{T}^{-}
\end{array}\right)
$$

where $\boldsymbol{T}^{+}\left(2 N_{1} \times 2 N_{1}\right), \boldsymbol{R}^{+}\left(N_{1} \times 2 N_{1}\right), \boldsymbol{T}^{-}\left(N_{1} \times N_{1}\right)$ and $\boldsymbol{R}^{-}\left(2 N_{1} \times N_{1}\right)$ are transmission and reflection matrices with and against the mean flow.

\subsection{Modal scattering of several cells}

The scattering matrix for two adjacent cells can be obtained from the scattering matrices of the single cells $\boldsymbol{S}^{(1)}$ and $\boldsymbol{S}^{(2)}$ by:

$$
\boldsymbol{S}^{(1+2)}=\left(\begin{array}{cc}
\boldsymbol{T}^{+(2)} \boldsymbol{E} \boldsymbol{T}^{+(1)} & \boldsymbol{R}^{-(2)}+\boldsymbol{T}^{+(2)} \boldsymbol{R}^{-(1)} \boldsymbol{F} \boldsymbol{T}^{-(2)} \\
\boldsymbol{R}^{+(1)}+\boldsymbol{T}^{-(1)} \boldsymbol{R}^{+(2)} \boldsymbol{E} \boldsymbol{T}^{+(1)} & \boldsymbol{T}^{-(1)} \boldsymbol{F} \boldsymbol{T}^{-(2)}
\end{array}\right),
$$

where

$$
\begin{aligned}
& \boldsymbol{E}=\left(\boldsymbol{I}-\boldsymbol{R}^{-(1)} \boldsymbol{R}^{+(2)}\right)^{-1}, \\
& \boldsymbol{F}=\left(\boldsymbol{I}-\boldsymbol{R}^{+(2)} \boldsymbol{R}^{-(1)}\right)^{-1} .
\end{aligned}
$$

The above iterative scattering matrix algorithm is used to obtain the scattering matrix of the periodic system shown in figure 1 .

\subsection{Bloch modes of the periodic system}

Since the governing equations, the boundary conditions, and the geometry are $W$ periodic along $x$ (where $W=L+S$ is the period), the Floquet-Bloch theorem states that any quantities $\phi(x, y)$ can be written as $\phi(x, y)=\phi_{B}(x, y) \mathrm{e}^{-\mathrm{i} k_{B} x}$, meaning that $\phi(x, y)$ is split into a field $\phi_{B}(x, y)$ which is $W$-periodic along $x$ superimposed to an exponential part in the $x$-direction involving only the Bloch wavenumber $k_{B}$ (Bradley 1994; Nennig et al. 2012; Schmid et al. 2017). This decomposition leads to $\phi(x+W, y)=$ $\phi_{B}(x+W, y) \mathrm{e}^{-\mathrm{i} k_{B}(x+W)}=\phi_{B}(x, y) \mathrm{e}^{-\mathrm{i} k_{B}(x+W)}=\phi(x, y) \mathrm{e}^{-\mathrm{i} k_{B} W}$. On the upstream and downstream boundaries of a unit cell, $x=0$ and $x=W$ for example, the relation between the qualities is $\phi(W, y)=\phi(0, y) \mathrm{e}^{-\mathrm{i} k_{B} W}$, which can be written in a vectorial form:

$$
\left(\begin{array}{l}
\boldsymbol{C}_{2}^{+} \\
\boldsymbol{C}_{2}^{-}
\end{array}\right)=\mathrm{e}^{-\mathrm{i} k_{B} W}\left(\begin{array}{l}
\boldsymbol{C}_{1}^{+} \\
\boldsymbol{C}_{1}^{-}
\end{array}\right)
$$

The scattering relation given by the equation (2.9) can be rewritten as,

$$
\boldsymbol{M}_{1}\left(\begin{array}{c}
\boldsymbol{C}_{1}^{+} \\
\boldsymbol{C}_{1}^{-}
\end{array}\right)=\boldsymbol{M}_{2}\left(\begin{array}{c}
\boldsymbol{C}_{2}^{+} \\
\boldsymbol{C}_{2}^{-}
\end{array}\right)
$$

where

$$
\boldsymbol{M}_{1}=\left(\begin{array}{cc}
\boldsymbol{T}^{+} & \boldsymbol{0} \\
-\boldsymbol{R}^{+} & \boldsymbol{I}
\end{array}\right), \quad \boldsymbol{M}_{2}=\left(\begin{array}{cc}
\boldsymbol{I} & -\boldsymbol{R}^{-} \\
\boldsymbol{0} & \boldsymbol{T}^{-}
\end{array}\right)
$$

Using equations (2.11) and (2.12), a generalized eigenvalue problem for a unit cell of the liner is found:

$$
\boldsymbol{M}_{1}\left(\begin{array}{c}
\boldsymbol{C}_{1}^{+} \\
\boldsymbol{C}_{1}^{-}
\end{array}\right)=\mathrm{e}^{-\mathrm{i} k_{B} W} \boldsymbol{M}_{2}\left(\begin{array}{c}
\boldsymbol{C}_{1}^{+} \\
\boldsymbol{C}_{1}^{-}
\end{array}\right),
$$

The eigenvalue problem (2.13) gives the Bloch wavenumbers $k_{B}$. Each eigenvector contains the coefficients of the modes solved in (2.7), the combination of which gives a Bloch mode of the cell. 

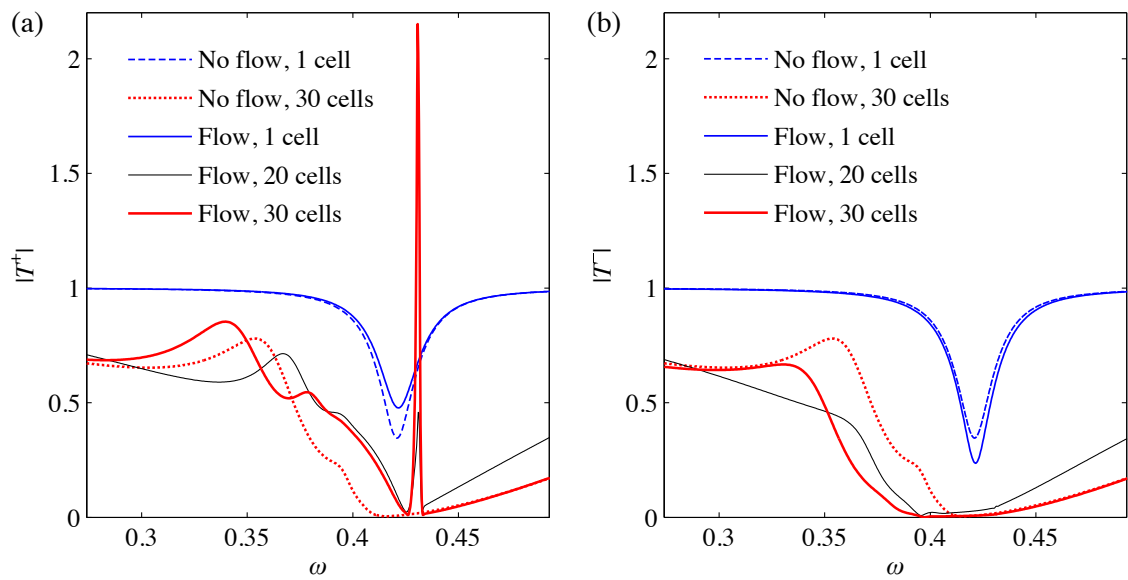

Figure 2. (Colour online) Transmission coefficients in (a) and against (b) the flow direction. The peak of $\left|T^{+}\right|$for the liner with 30 cells occurs at $\omega=0.4310(1573 \mathrm{~Hz})$.

\section{Results}

Calculations in the following are carried out on an array of $2 \mathrm{D}$ cells sketched in figure 1. Each cell consists of a deep narrow cavity attached to a short duct containing a mean shear flow. The geometrical parameters are: $H^{*}=15 \mathrm{~mm}, D^{*}=55 \mathrm{~mm}, L^{*}=1 \mathrm{~mm}$, and $S^{*}=0.2 \mathrm{~mm}$. The Mach number averaged over the cross-section is $M_{0}=0.1$ and the velocity profile is given by a simple polynomial law with a unity average value,

$$
f=f_{0}\left(1-y^{m}\right) \quad \text { with } f_{0}=\frac{m+1}{m},
$$

where the parameter $m=10$ is used in the present calculations. At the mouth of each cavity a thin sheet with a normalized resistance $R=0.0175$ has been added. This value has been empirically chosen such as the global cell instability exists (see discussion of figure 7) while the unstable modes over the cavities are suppressed. The number of the discrete points in the duct is 600 , so 1800 transverse modes are solved from (2.7) in the duct.

\subsection{Instability near the acoustic resonance}

The transmission coefficients for a plane wave propagating through one and several cells in the $\pm x$-directions are shown in figure 2 . Without flow, $\left|T^{ \pm}\right|$of a single cell shows a minimum at the resonance frequency. This minimum is larger than zero because of the resistive sheet. The liner with 30 cells stops the sound transmission more effectively at resonance than a single cell. With flow, minimums of $\left|T^{ \pm}\right|$around resonance still happen for one or several cells. The symmetry of sound propagation in the $\pm x$-directions, however, is broken by the convective effect and the flow-acoustic interaction near the liner. More interestingly, a narrow hump is observed in $\left|T^{+}\right|$with flow. Especially, the amplitude can be larger than unity, which means the incident plane sound wave is amplified as it passes through the periodic cells. Figure 3 shows the pressure fields in the duct-liner system for a plane wave incidence from the upstream duct. At the peak frequency of the hump shown in figure 2(a), a surface wave excited by the sound wave at the upstream part of the liner is observed in figure 3(b). It convectively grows over the liner and regenerates sound near the downstream edge of the liner. In this case, the incident plane wave is transformed 
(a)

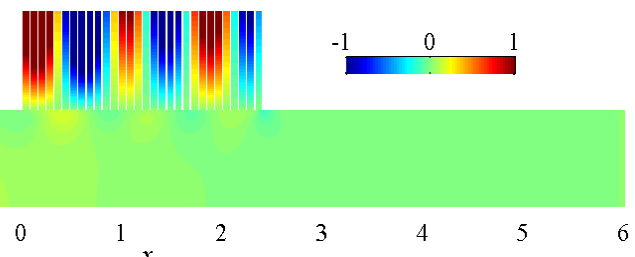

(b)

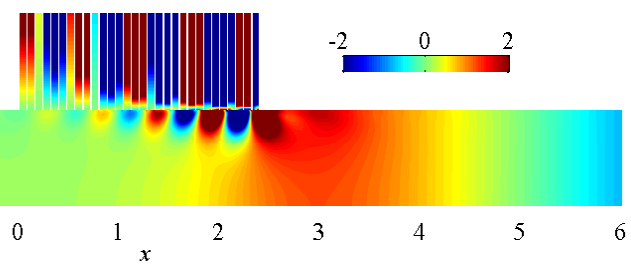

Figure 3. (Colour online) Iso-colour plots of the real part of the pressure for a plane sound wave incidence at frequencies (a) $\omega=0.4266(1557 \mathrm{~Hz})$ and (b) $\omega=0.4310(1573 \mathrm{~Hz})$. Note that the fields are only plotted for the duct and part of the liner cavities.

into an unstable hydrodynamic wave that is amplified as it propagates though the lined segment. At the downstream edge, this hydrodynamic wave generates an acoustic wave whose level is higher than the incident level. At a slightly lower frequency, $\omega=0.4266$ $(1557 \mathrm{~Hz})$, figure 3(a) also shows a surface wave over the liner. However, it decreases along the liner and no sound amplification is observed. So, the incident sound wave is stopped by the liner due to acoustic resonance. The results of the fields over the surface of the liner bear interesting resemblance to PIV experiments (Marx et al. 2010). Such convectively growing surface wave and the associated sound amplification, first observed in experiments, were attributed to hydrodynamic instabilities (Ronneberger \& Jüschke 2007; Aurégan \& Leroux 2008; Marx et al. 2010).

To explore the root cause of such an instability, the shear layer instability in zone II is first examined by looking the wavenumbers of the acoustic and hydrodynamic modes deduced from (2.7). With a shear flow, in addition to the classical modes, neutral hydrodynamic modes (Brambley et al. 2012) appear. They result from the singularities of the Pridmore-Brown equation (Pridmore-Brown 1958) when $\omega-M k=0$, thus they form a continuous hydrodynamic spectrum on the real axis, $\omega / M_{\max }<k<\omega / M_{\min }$, where $M$ is the non-dimensional mean flow velocity. Under certain conditions, an unstable hydrodynamic mode can occur, which is known as the Kelvin-Helmholtz instability (Schmid \& Henningson 2000). The Briggs-Bers causality criterion is used to distinguish an unstable hydrodynamic mode from an acoustic mode decaying in the $-x$ direction (Briggs 1964; Bers 1983). For that, in the $\exp (i(\omega t-k x))$ convention, a negative imaginary part is added to the frequency $\omega$ and the modes with wavenumbers that are in the lower complex plane when $\operatorname{Im}(\omega) \rightarrow-\infty$ propagate in the $+x$ direction, while the modes with wavenumbers that are in the upper complex plane when $\operatorname{Im}(\omega) \rightarrow-\infty$ propagate in the $-x$ direction. The Briggs-Bers criterion states that, if one of the mode crosses the real axis while the imaginary part of the frequency $\operatorname{Im}(\omega)$ ranges from $-\infty$ to 0 , it means that this mode is convectively unstable. The wavenumbers of the acoustic and hydrodynamic modes in zone II are plotted by circles in figure 4 when the frequency is real. It can be noted that the continuous line of hydrodynamic modes on the real axis has been transformed into a set of points because of the discretization of the problem. When the resistance $R=0$, see figure 4 (a), one unstable mode with a high amplification rate appears. This unstable mode is stabilized when a resistive sheet is added, as shown 

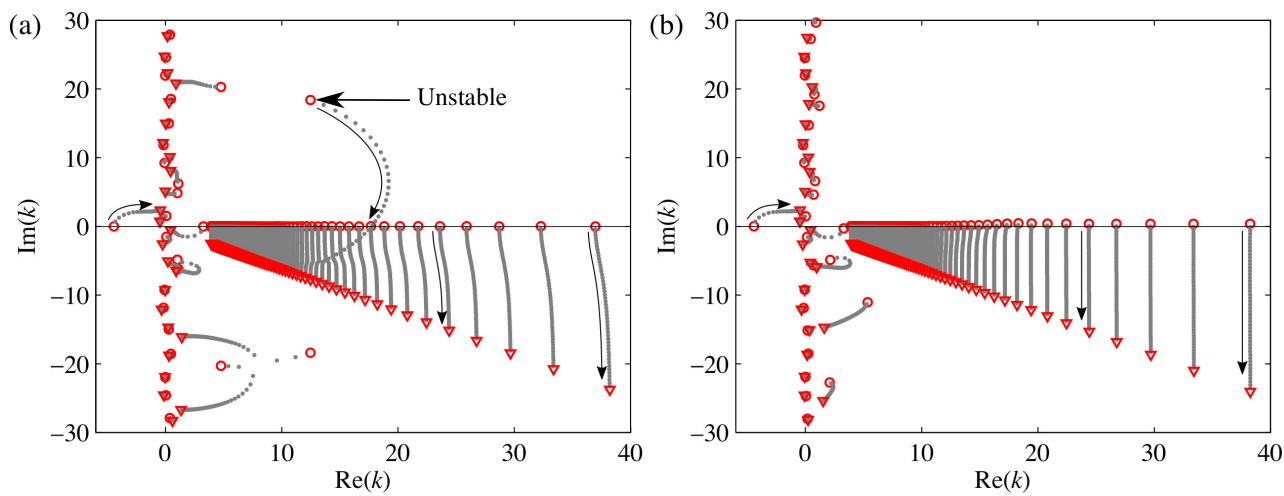

FiguRE 4. (Colour online) Tracing of the eigenvalues in zone II: $\operatorname{Re}(\omega)$ is kept constant 0.4310 $(1573 \mathrm{~Hz}$ ) while $\operatorname{Im}(\omega)$ runs from 0 (results are denoted by the circles) to -0.2740 (results are denoted by the triangles). (a) No resistive sheet and (b) a resistive sheet $R=0.0175$ is introduced at the opening of the cavity. The small arrows indicate the direction of the variation of the eigenvalues for the increasing negative part of $\omega$.

in figure 4 (b). It should be noted that the unstable mode is gradually stabilized by increasing $R$. However, it can be observed that several neutral modes are now slightly out of the real axis. This effect is due to the discretization and those modes tend to the real axis when the number of discretization points is increased. To avoid this numerical instability and to mimic the effect of turbulence that dissipates quickly the coherent hydrodynamic waves, an artificial damping is added to all the hydrodynamic modes by adding to their wavenumbers $k_{h}$ a negative imaginary part $-\mathrm{i} \epsilon \operatorname{Re}\left(k_{h}\right)$. The effect of this artificial damping on the sound amplification is presented in figure 11(a) of the Appendix A. For all the results presented in this paper (except on figures 4 and 10 where $\epsilon=0$ and on figure 11 where the effect of $\epsilon$ is studied), a damping with $\epsilon=0.06$ was added to the hydrodynamic modes in zones I and II. This value has been empirically chosen to be the smallest value that stabilizes all the discrete hydrodynamic modes, in the frequency range of sound amplification shown in figure $2(\mathrm{a})$.

The aeroacoustic behaviour of one cell, consisting of zones I and II, can be determined by the Bloch wavenumbers $k_{B}$ which are obtained from the eigenvalues problem (2.13). Note that in the calculations of $\left|T^{ \pm}\right|$in this paper the wave reflection by the sudden changes from hard to lined to hard duct is included, whereas in Bloch mode analysis, an infinitely long periodic structure is considered. Figure 5(a) gives the variation of $k_{B}$ when the frequency goes from $\omega=0.2740$ to 0.4932 . It can be seen that one of the cell's hydrodynamic modes is emerging from the line of attenuated hydrodynamic modes and it is passing through the real axis to become amplified. This unstable Bloch mode is observed in a very limited range of frequencies $(0.4277<\omega<0.4332)$ near the quarter wavelength $(\lambda / 4)$ resonance of the cavity. The maximum amplification rate of the Bloch unstable mode, i.e. the maximum in the imaginary part of $k_{B}^{u}$, happens at the same frequency as the peak of $\left|T^{+}\right|$for 30 cells in figure 2 . The relative errors of the calculations are shown in figure $5(\mathrm{~b})$, where the errors are defined as $\mid k_{B}^{u}\left(N_{1}\right)-$ $k_{B}^{u}\left(N_{1 R}\right)|/| k_{B}^{u}\left(N_{1 R}\right) \mid$ with the reference result computed with $N_{1 R}=1920$ (same for $T^{ \pm}$). It is interesting to note that even when all the transverse modes are stable in zones I and II (the hydrodynamic modes have been stabilized by the artificial damping in zones I and II), a cell instability can still occur. It should also be noted that the upstreampropagating acoustic wave identified by $k_{B 0}^{-}$is strongly attenuated when the instability 

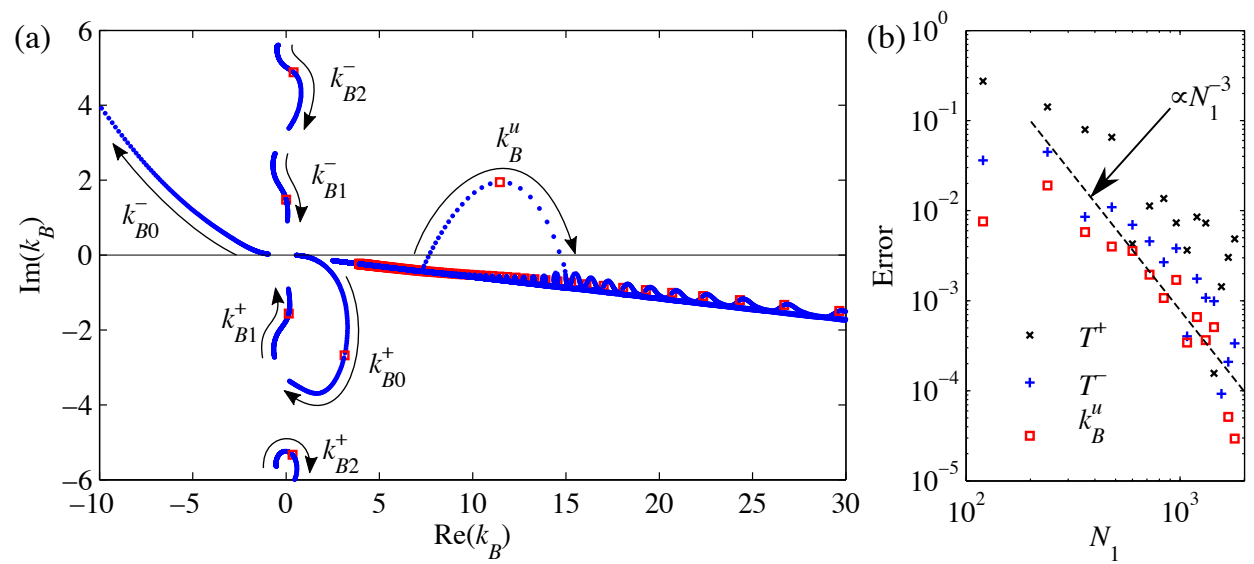

Figure 5. (Colour online) (a) Bloch wavenumbers $k_{B}$ of one cell for frequency sweep from $\omega=0.2740$ to $0.4932(1000-1800 \mathrm{~Hz})$. The arrows in (a) denote the direction of the variation of the modes with increasing frequency. The red squares indicate the $k_{B}$ at frequency $\omega=0.4310$ $\left(1573 \mathrm{~Hz}\right.$ ), which is the peak frequency of $\left|T^{+}\right|$for 30 cells in figure 2. (b) The relative errors of $\left|k_{B}^{\mathrm{u}}\right|$ and $\left|T^{ \pm}\right|$for 30 cells at frequency $\omega=0.4310(1573 \mathrm{~Hz})$.

occurs $\left(k_{B 0}^{-}=-17.56+100.8 \mathrm{i}\right.$ at frequency $\left.\omega=0.4310\right)$. This means that the global instability that consists of the unstable hydrodynamic wave and the first left-running acoustic mode described in lined duct by Pascal et al. (2017) does not occur in our system.

Figure 6(a) and (b) show the periodic fields for $\omega=0.4310$ when the unstable Bloch wave propagates through the lined duct with the maximum amplification. This unstable Bloch mode presents a sharp peak of vorticity at $y_{c}=0.9590$. At this position the mean velocity is $M_{0} f\left(y_{c}\right)=0.03763$ that is equal to the wave velocity that can be computed from the wavenumber $k_{B}^{u}=11.453+1.949 \mathrm{i}$. This indicates that this wave is convected by the mean flow and it could be called a hydrodynamic unstable mode.

Some inhomogeneous effects in the $x$-direction are present very close to the mouths of the cavities $(y>0.98)$ and they are shown by the black thin lines in figure 6 (c) and (d). Nevertheless, at vertical position $y=y_{c}$ the quantities displayed by the red thick lines are nearly constant. It means that, except very near the wall, the periodic field of the unstable mode is nearly independent of $x$ and that any field can be written with a transverse mode form: $\phi(x, y)=\phi_{T}(y) \mathrm{e}^{-\mathrm{i} k_{B} x}$. In this case, (2.5) and (2.6) can be easily solved using a new variable $Y$, such as $\mathrm{d} P / \mathrm{d} y=Y P$, whose variations are given by

$$
\frac{\mathrm{d} Y}{\mathrm{~d} y}=-Y^{2}-\frac{2 M_{0} k_{B}}{\omega-M_{0} f k_{B}} \frac{\mathrm{d} f}{\mathrm{~d} y} Y-\left(\omega-M_{0} k_{B} f\right)^{2}+k_{B}^{2} .
$$

The equation (3.2) can be integrated from $y=0$ where $Y=0$ to $y=1$. When $Y$ is known, the pressure $P$ can be found by integrating $\mathrm{d} P / \mathrm{d} y=Y P$ and all the other variables can be deduced from those values. On figure 6 (e) and (f), the values obtained by this integration is favourably compared to the values obtained by the Bloch method. This approximated method helps to understand the coupling of this hydrodynamic mode with the $\lambda / 4$ resonance of the cavity. The value of $Y$ at the wall is directly linked to an equivalent impedance of the wall by $Z_{w}=-\mathrm{i} \omega / Y(1)$. For $\omega=0.4310$ and $k_{B}=k_{B}^{u}$, the impedance is $Z_{w}^{u}=0.0219+0.0132 \mathrm{i}$ which can be compared to the impedance without flow of the cavity $Z_{m}=(W / L)(R-\mathrm{i} / \tan (\omega D))=0.0210+0.0113$ i. Thus $Z_{w}$ (which 

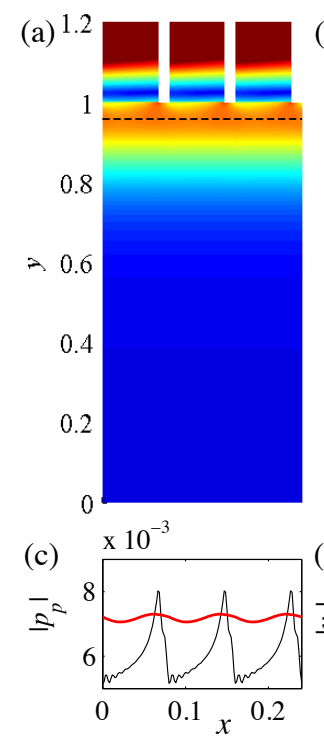

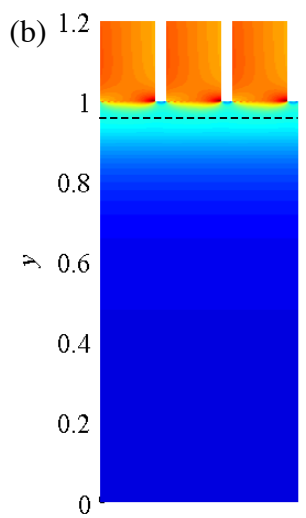

(d) 0.4

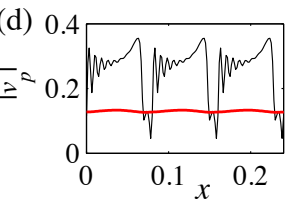

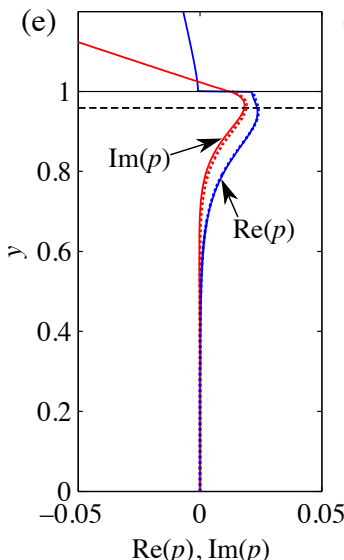

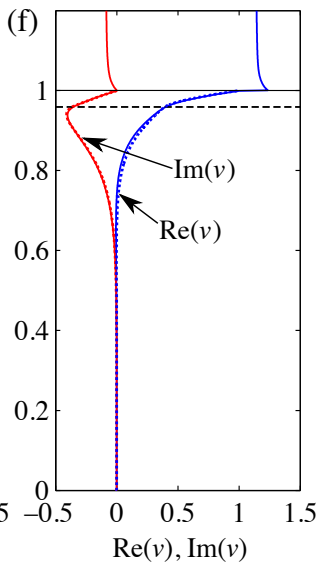

Figure 6. (Colour online) Iso-colour plots of the amplitude of the periodic part of (a) pressure and (b) $v$-velocity when the unstable Bloch mode propagates in the lined duct. (c) and (d) show respectively the amplitudes of the periodic part of pressure and $v$-velocity as a function of $x$, the lines denote quantities at 2 different $y$-positions: black thin lines for $y=1-\Delta h / 2$, and red thick lines for $y=0.9590$. (e) and (f) present the comparison of the periodic part of $p$ and $v$-velocity averaged along $x$ on one cell (solid lines) and obtained from (3.2) (dashed lines). The horizontal dashed lines represent the $y$-position of the peak of vorticity $\left(y=y_{c}=0.9590\right)$, where the mean flow velocity equals to the wave velocity of $k_{B}^{u}$. The calculations are made at frequency $\omega=0.4310$. Note that the fields are only plotted for the duct and part of the liner cavities. Also note that the short-wavelength oscillations very close to $y=1$ observed in (d) are due to the spurious numerical modes caused by the discontinuity at the interface between the two zones at $y=1$ (same in figure $9(\mathrm{~d})$ ).

only depends, at given frequency and wavenumber, on the Mach number and on the shape of the profile) is a reasonable approximation of the impedance that must be put on the wall to sustain an unstable hydrodynamic mode. By studying the variation of $Z_{w}$ when the wavenumber $k_{B}$ is slightly varied around the value obtained by the Bloch computation $k_{B}^{u}$ (see figure 7 ), it can be seen that an increase in the resistance $R$ induces a decrease of the amplification $\operatorname{Im}\left(k_{B}\right)$ and that for each frequency there is a maximum resistance over which no unstable mode can exist. In the present case, this maximum resistance is 0.0285 . This maximum resistance is always very small and that is why in most of the practical applications where the dissipation is much higher, this instability does not appear. One way of increasing the wall resistance is to increase the spacing $S$ between the cavities so that the percentage of open area is decreased. The effect of $S$ on the sound amplification is presented in figure 11 (b) of the Appendix A. It is shown that an increase in $S$, i.e. an increase in the equivalent wall resistance, can mitigate or suppress the sound amplification. The imaginary part of $Z_{w}$ is also small compared to 1 and always positive around $k_{B}^{u}$. With a cavity or a Helmholtz resonator this kind of small values can only be obtained near the resonance of the wall $(p \simeq 0)$. The positive value of the imaginary part of $Z_{w}$ means that instability occurs for frequencies slightly higher than the resonance frequency because the imaginary part of the impedance of a cavity always increases from $-\infty$ when $\omega \rightarrow 0$ and being 0 at the wall resonance. 

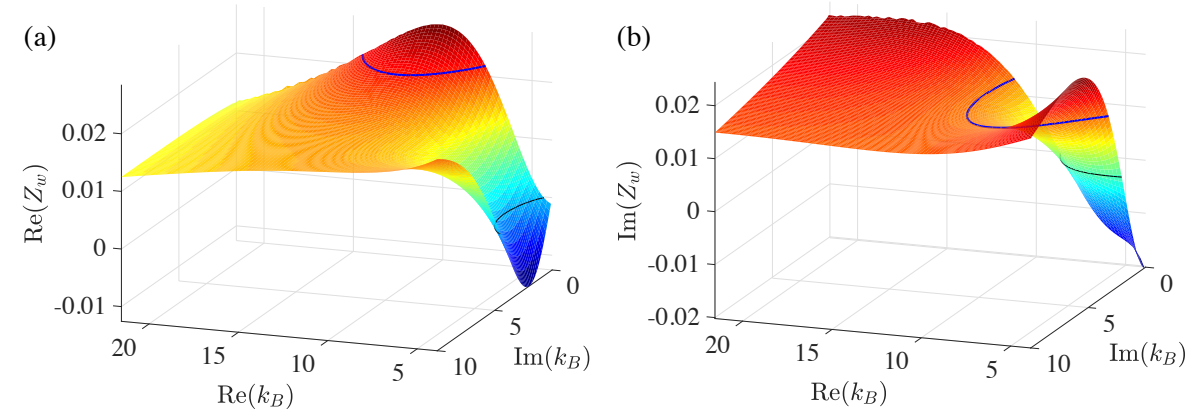

Figure 7. Real (a) and imaginary (b) parts of the wall impedance $Z_{w}$ that is computed using (3.2) for $\omega=0.4310$. The blue thick lines correspond to $Z_{w}=Z_{w}^{u}$ and the black thin lines to $Z_{w}=0$.

The above analysis using (3.2) is valid here because the vorticity of the mode is concentrated far from the wall. The results can differ if this vorticity mode interact strongly with the axial non-uniformities due to the sidewalls of the cavities. In this case the full Bloch analysis cannot be simplified.

The instability occurring in this system of small cavities is then due to a vorticity mode that is regularly amplified by the resonance of the cavities and it transfers some energy from the mean flow to the perturbations. This mechanism is quite different from those which cause whistling or amplification of sound in cavities, side branches, corrugated pipes, or Helmholtz resonators studied by Tam \& Block (1978), Ziada \& Shine (1999), Nakiboglu et al. (2011, 2012), Nakiboglu \& Hirschberg (2012), Yamouni et al. (2013), Dai et al. (2015), and Golliard et al. (2016). Ziada \& Shine (1999) and Nakiboglu \& Hirschberg (2012) studied the hydrodynamic interference between multiple side branches or cavities and its effect on the Strouhal number. Sound amplification in those situations occurs when the length of the cavity opening is approximately equal to an integer multiplied by the unstable wavelength thus the Strouhal number $S r_{c}=\omega L /\left(2 \pi M_{c}\right)$ is around $1,2,3, \ldots$, where $M_{c}$ is the convection velocity of the instability wave. For the present case, $M_{c}$ calculated from the real part of the Bloch wavenumber at the peak amplification is $0.3763 M_{0}$, which gives us $S r_{c}=0.1215$ and the wavelength of the unstable mode is not linked to the length of a single cavity or cell (here this wavelength is about 7 times the length of a cell).

It can also be noted that along the system of 30 cells computed for figures 2 and 3, the unstable Bloch mode is amplified by a factor of more than 80 while the acoustic wave is amplified only by a factor of about 2 . This means that the transfers from the incident plane acoustic mode to the hydrodynamic unstable mode and from the hydrodynamic unstable mode to the transmitted plane acoustic mode are rather inefficient.

\subsection{Bloch mode stabilization by using cavities with different depths}

We know from the previous experiments (Aurégan \& Leroux 2008; Marx \& Aurégan 2013) that the hydrodynamic instability over a liner that consists of identical cavities can be suppressed by increasing the damping of the system or by increasing the spacing between cavities. The effects of these parameters on such an instability are qualitatively replicated in the previous subsection, where the validity of the homogenized approach has also been verified. In this subsection, we examine a liner that consists of different cavities.

Starting from the case studied in section 3.1, we slightly increase the depth of one 

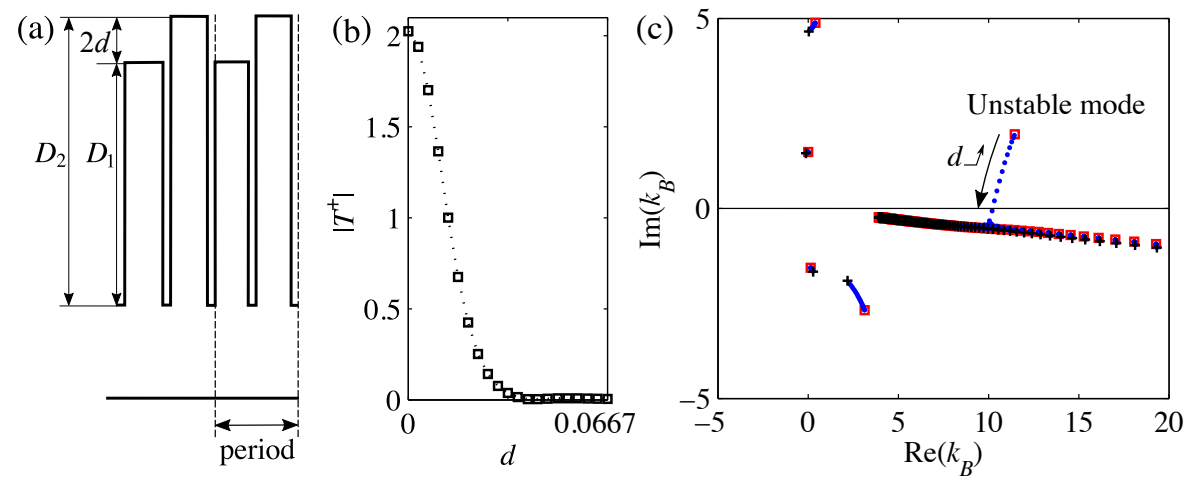

FIGURE 8. (Colour online) Flow-acoustic coupling in a liner with 15 periodic cells, each of which consists of two cavities with different depths: $D_{1}=D-d$ and $D_{2}=D+d$, as sketched in (a). (b) presents $\left|T^{+}\right|$for a plane wave incidence to the liner as a function of $d$. (c) shows the variation of the Bloch wavenumbers $k_{B}$ for $d$ increasing from 0 to $0.0667\left(d^{*}=1 \mathrm{~mm}\right)$. In (c), the arrow indicates the direction of the variation of $k_{B}$ for the increasing $d$, the red squares denote $k_{B}$ when $d=0$, and the black crosses denote $k_{B}$ when $d=0.0667$. The calculations are made at frequency $\omega=0.4310$.
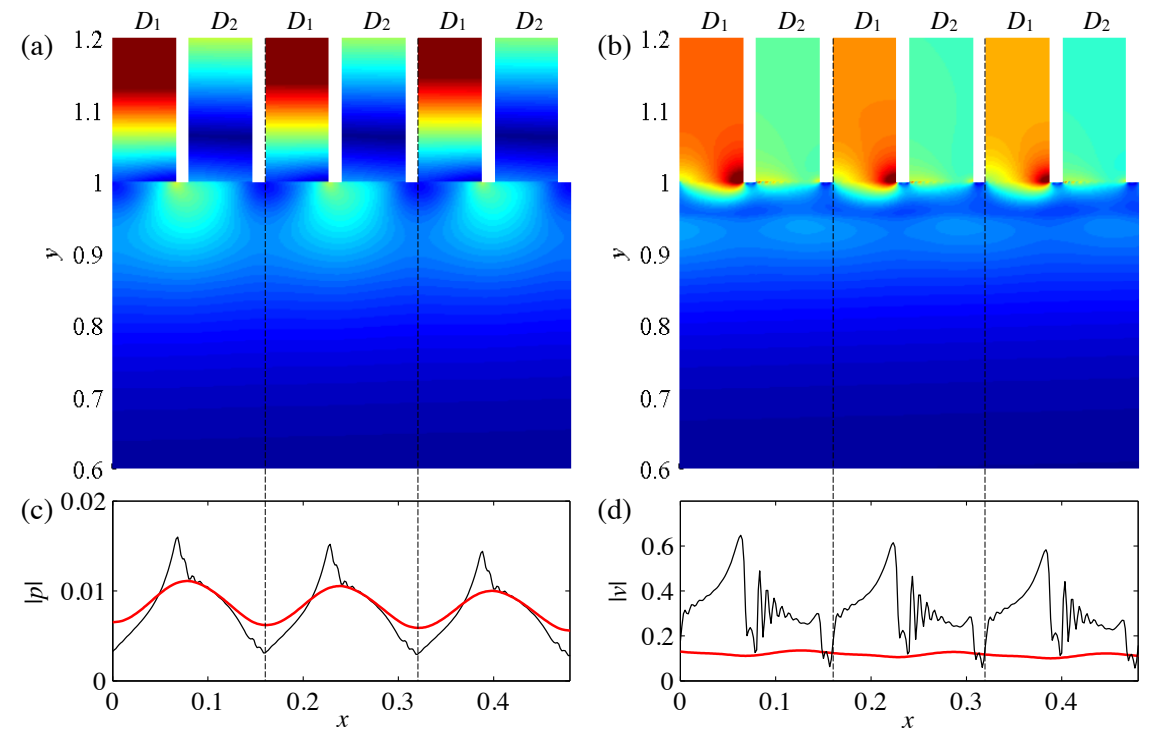

Figure 9. (Colour online) Iso-colour plots of the amplitudes of (a) pressure and (b) $v$-velocity for 3 periodic cells when the Bloch mode $\left(k_{B}=10.056-0.3252 \mathrm{i}\right)$ propagates in the lined duct. The vertical dashed lines denote the boundaries of the periodic cells. Each cell consists of two cavities with different depths: $D_{1}=D-d$ and $D_{2}=D+d$, where $d=0.04\left(d^{*}=0.6 \mathrm{~mm}\right)$. (c) and (d) show the amplitudes of pressure and $v$-velocity as a function of $x$ at 2 different $y$-positions: black thin lines for $y=1-\Delta h / 2$, and red thick lines for $y=0.9525$ where the mean flow velocity equals to the wave velocity of $k_{B}$. The calculations are made at frequency $\omega=0.4310$. Note that the fields are only plotted for part of the duct and the liner cavities.

cavity out of two and slightly decrease the depth of the other cavity by the same amount so that the average remains the same, see figure $8(\mathrm{a})$. Thus, we consider 15 periodic cells, each of which consists of two cavities of different depths. The sound amplification is progressively suppressed by increasing the difference $d$ between the depths of the 

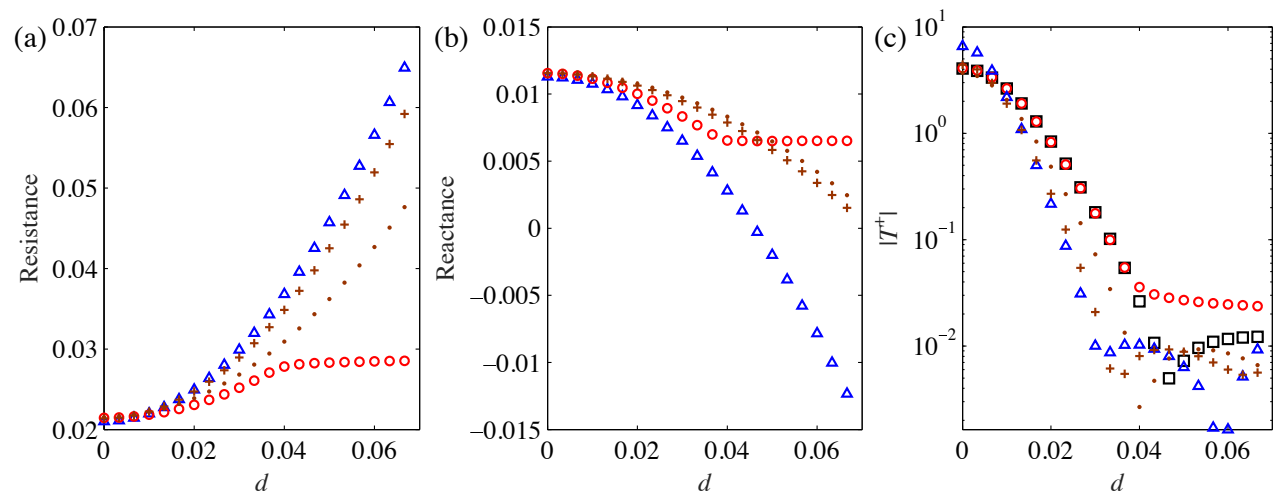

Figure 10. (Colour online) Educed resistance (a) and reactance (b) from the Bloch modes, as a function of $d$ for the liner sketched in figure 8(a). (c) Comparison of the transmission coefficients obtained by the Bloch and the homogenized approaches. In (a) and (b), circles, crosses and dots are impedances respectively educed from the Bloch modes $k_{B}^{u}, k_{B 1}^{+}$and $k_{B 1}^{-}$using (3.2), triangles denote $Z_{a v}$. In (c), squares denote the Bloch approach, circles, crosses, dots, and triangles denote the homogenized approach with wall impedances educed from $k_{B}^{u}, k_{B 1}^{+}, k_{B 1}^{-}$, and given by $Z_{a v}$ respectively. The calculations are made at frequency $\omega=0.4310$. Note that no damping is added to the hydrodynamic modes in the calculation of this figure, i.e. $\epsilon=0$.

two cavities, see figure 8(b). A very small difference in depth (around $1 \%$ ) completely destroys the instability. The Bloch fields of the amplitudes of $p$ and $v$ for 3 periodic cells when $d=0.04$ are presented in figure 9 . The chosen Bloch mode, with a wavenumber $k_{B}=10.056-0.3252 \mathrm{i}$, is the one that is progressively stabilized by increasing $d$, see figure $8(\mathrm{c})$. The periodical decrease of the disturbances from cell to cell is demonstrated in the pressure and the velocity fields. It is also shown that the hydrodynamic disturbances grow when passing the smaller cavity, but reduce in the segment with the deeper cavity. In the case of identical cavities, the phase relation between the vorticity and the vertical velocity is such that some energy can be transferred from the mean flow to the perturbations. In the two depths case, there is a phase difference in the vertical velocities from two adjacent tubes. Near the resonance, this phase difference increases quickly when the depth changes. Therefore, the energy transfer can no longer be positive for the two adjacent cavities.

In figure 10 the homogenized results are compared to the complete Bloch analysis. For this, we first used the Bloch mode wave numbers to compute the homogeneous impedances by integrating (3.2). In figure 10 (a) and (b), the real and imaginary part of the impedance obtained from the unstable hydrodynamic Bloch mode $k_{B}^{u}$ (circles) and the least attenuated acoustic modes in the $\pm x$-directions $k_{B 1}^{ \pm}$(crosses and points) are presented. They are close to each other for $d<0.04$. For $d>0.04$, the impedance deducted from $k_{B}^{u}$ becomes almost constant but we can note that, for these $d$ values, the mode reaches the hydrodynamic continuum where the wave numbers no longer depend on impedance. The impedance values deduced are therefore questionable. On the same figures, the educed impedances are compared to the average impedance (triangles) without flow over one cell: $Z_{a v}=2 W / L /\left(1 / Z_{1}+1 / Z_{2}\right)$, where $Z_{1}=R-\mathrm{i} / \tan \left(\omega D_{1}\right)$ and $Z_{2}=R-\mathrm{i} / \tan \left(\omega D_{2}\right)$. The predicted and educed resistances correspond reasonably well for $d<0.04$. There is a larger difference in reactance. When $d=0$, the velocity is almost the same in each successive cavity. When $d$ increases, there is a velocity difference between successive cavities and part of the fluid passes by turning from one cavity to the next. This results in an additional effective mass that could explain the difference between predicted and induced reactances. The transmission coefficient calculated using 
the impedance educed from $k_{B}^{u}$ is shown in figure 10 (c) and it is compared to the full Bloch approach. It indicates that the homogenized approach approximates to the full Bloch analysis when the impedance educed from unstable Bloch mode is used to describe the lined wall. The good agreement of the results in the unstable regime shows that the homogenized approach is a very good first approximation for the unstable behaviour over a liner even if the tubes are of different heights. The disappearance of the unstable mode when $d$ increases is therefore simply related to the increase in equivalent resistance which is in line with the results in figure $7(\mathrm{a})$. Note that the average impedance $Z_{a v}$ and the impedances educed from $k_{B 1}^{+}$and $k_{B 1}^{-}$can also predict the trend of stabilization by the increasing $d$. However, a large discrepancy in the calculated transmission coefficients is observed even though $Z_{a v}$ is very close to the educed impedance when $d$ is close to zero. For $d=0$, the difference between $Z_{a v}$ and the educed impedance is $1.9 \%$ for resistance and $2.6 \%$ for reactance. This difference induces a $7 \%$ change in the amplification $\operatorname{Im}\left(k_{B}^{u}\right)$ and, due to the exponential amplification, a $61 \%$ increase in the transmission coefficient. This example shows the very large sensitivity of the peak in the transmission coefficient to a very precise description of the wall condition.

\section{Conclusion}

The aeroacoustic instability over a low resistance liner with a grazing flow is studied by a two-dimensional (2D) cavity-by-cavity approach using the multimodal method in combination with the Bloch theory. The mean shear flow over the liner is assumed unaltered in the streamwise direction and the acoustic and hydrodynamic disturbances are described by the linearized Euler equations with artificial damping. Such an instability was first observed in experiments and has been investigated by many homogenized methods based on an equivalent wall impedance.

We first consider a liner of 30 periodic cells, each of which consists of a deep narrow cavity attached to a short duct containing a mean shear flow. A sound amplification of the incident plane wave in the upstream duct is predicted by the present discrete model. The amplification happens in a very limited frequency range that is slightly higher than the resonance frequency of the cavities. At the amplification frequencies, a hydrodynamic surface wave is excited by the sound wave at the upstream part of the liner. After a convective growth over the liner, it regenerates sound near the downstream edge of the liner at a level higher than this incident level. The effects of an artificial damping and of the spacing between cavities on the sound amplification are estimated. The results obtained with this periodic analysis are very similar to the previous experimental results. Nevertheless, due to the large sensitivity of the instability to the relevant parameters, such as the dissipations and the longitudinal inhomogeneity of the mean flow, quantitative comparisons with experiments is rather difficult.

The liner instability is demonstrated by the present Bloch approach. From the variation of the Bloch wavenumbers as a function of frequency, we can see that one of the hydrodynamic modes emerges from the line of attenuated hydrodynamic modes and crosses the real axis to become amplified. This unstable Bloch mode appears in a very limited range of frequencies near the quarter wavelength $(\lambda / 4)$ resonance of the cavity. The maximum amplification rate of the Bloch unstable mode, i.e. the maximum in the imaginary part of the Bloch wavenumber, occurs at the same frequency as the maximum in the acoustic transmission coefficient. One of the interesting results of this analysis is to show that even when all the transverse modes are stable in the duct-cavity system (the hydrodynamic transverse modes have been stabilized by an artificial damping), a global cell instability can still occur. This means that the Kelvin-Helmholtz instability 
over the cavities is not a necessary condition for the hydrodynamic instability over the liner and the related sound amplification.

This instability is due to a vorticity mode that is regularly amplified by the resonance of the cavities and it transfers some energy from the mean flow. The unstable Bloch mode has a sharp peak of vorticity at a transverse position in the flow duct, where the mean velocity is equal to the wave velocity computed from the Bloch wavenumber. It means that this wave is convected by the mean flow and it could be called a hydrodynamic unstable mode. The periodic part of the pressure, velocity and vorticity at the position of the vorticity peak is nearly constant, which suggests that a homogenized method based on an equivalent wall impedance can be used to describe the behaviour of such unstable mode. The homogenized model reveals that a small wall resistance and a small and positive reactance are two necessary conditions for the liner instability.

In a case where each periodic cell consists of two cavities of different depths, it is shown that a small depth difference can completely suppress the unstable behaviour above the liner. A homogenized approach can also correctly model the hydrodynamic instability in this case and it reveals that for the Bloch mode stabilization, increasing the depth difference is equivalent to increasing the resistance of the lined wall.

\section{Acknowledgments}

Y.A. gratefully acknowledges support from the ANR international project FlowMatAc No. ANR-15-CE22-0016-01; X.D. also gratefully acknowledges support from the ANR international project FlowMatAc during his stay in France.

\section{Appendix A. Effects of the artificial damping and the spacing between cavities on sound amplification}

As mentioned in section 3.1, some artificial damping has been added to the hydrodynamic modes in the calculations. To analyze its effect on propagation, we calculated $\left|T^{+}\right|$ of 30 cells with the same geometry but for different dissipation rates on the hydrodynamic modes of zones I and II. It is shown in figure 11(a) that an increase in the dissipation factor $\epsilon$ of the hydrodynamic waves leads to a clear reduction in sound amplification. This effect has already been observed in Marx \& Aurégan (2013) and shows the importance of having dissipation to correctly predict the peak amplitude. Also, to verify that our model can explain the experimental observation that the peak is very sensitive to the percentage of open area (POA) (Aurégan \& Leroux 2008), the spacing between the cavities $S$ has been varied and the results are shown in figure 11(b). They show that the peak value is highly dependent on the POA (division by 20 when the spacing is doubled). This effect can be interpreted as being related to the increase in coating resistance with the decrease in POA in the continuous impedance approach (see figure 7).

\section{REFERENCES}

Alomar, A., \& AurÉgan, Y. 2017 Particle image velocimetry measurement of an instability wave over a porous wall in a duct with flow. Journal of Sound and Vibration 386, 208-224.

AurÉGAn, Y. \& Leroux, M. 2008 Experimental evidence of an instability along an impedance wall with flow. J. Sound Vib. 317, 432-439.

AurÉGAn, Y. \& Singh D.K. 2014 Experimental observation of a hydrodynamic mode in a flow duct with a porous material. J. Acoust. Soc. Am. 136, 567-572.

Aurégan, Y., Starobinski, R. \& Pagneux, V. 2001 Influence of grazing flow and dissipation 

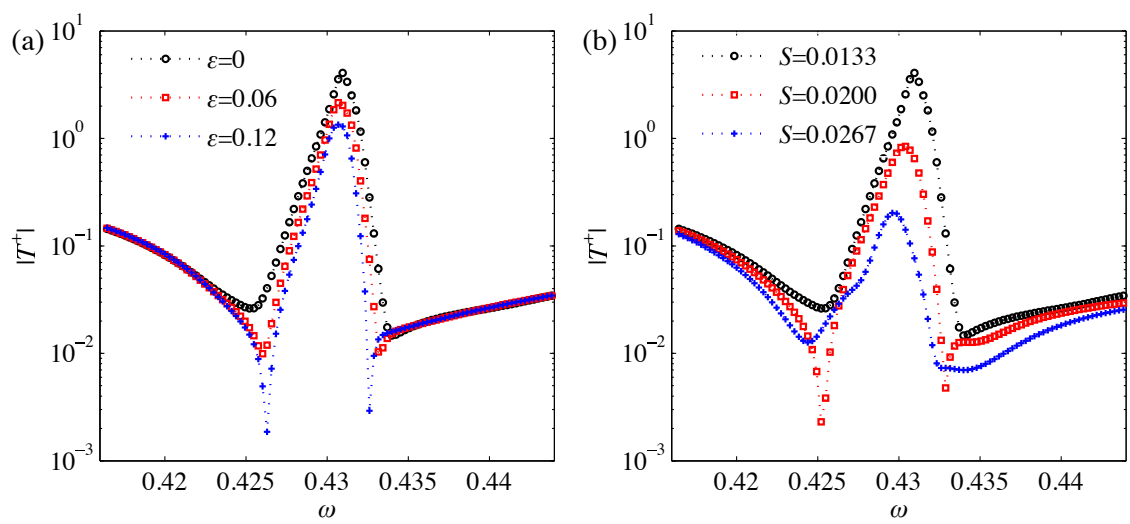

Figure 11. (Colour online) Effects of the dissipation of the hydrodynamic modes (a) and the length of spacing between the adjacent cavities (b) on the sound amplification in the periodic liner with 30 cells. In (a), the calculations are made with the default geometrical parameters but different artificial damping factor $\epsilon$. In (b), $\epsilon=0$, while the length of spacing $S$ is varied.

effects on the acoustic boundary conditions at a lined wall. J. Acoust. Soc. Am. 109, 59-64.

Bers, A. 1983 Space-time evolution of plasma instabilities - absolute and convective. In Basic Plasma Physics, Handbook of Plasma Physics (ed. A. A. Galeev \& R. N. Sudan), vol. 1, pp. 451-517. North-Holland.

Bradley, C. E. 1994 Time harmonic acoustic Bloch wave propagation in periodic waveguides. Part I. theory. J. Acoust. Soc. Am. 96, 1844-1853.

Brambley, E.J. 2009 Fundamental problems with the model of uniform flow over acoustic linings. J. Sound Vib. 322, 1026-1037.

Brambley, E.J. 2011 A well-posed boundary condition for acoustic liners in straight ducts with flow. AIAA J. 49, 1272-1282.

Brambley, E. J., Darau, M. \& Rienstra, S. W. 2012 The critical layer in linear-shear boundary layers over acoustic linings. J. Fluid Mech. 710, 545-568.

Brambley, E.J. \& Peake, N. 2006 Classification of aeroacoustically relevant surface modes in cylindrical lined ducts. Wave Motion 43, 301-310.

Brandes, M. \& Ronneberger D. 1995 Sound amplification in flow ducts lined with a periodic sequence of resonators. AIAA peper 95-126, pp. 893-901.

Briggs, R. J. 1964 Electron-Stream Interaction with Plasmas, MIT.

DAI, X. \& AurÉgan, Y. 2016 Acoustic of a perforated liner with grazing flow: Floquet-Bloch periodical approach versus impedance continuous approach. J. Acoust. Soc. Am. 140, $2047-2055$.

DAI, X., Jing, X. \& Sun, X. 2015 Flow-excited acoustic resonance of a Helmholtz resonator: Discrete vortex model compared to experiments. Phys. Fluids 27, 057102.

GABARD, G. 2013 A comparison of impedance boundary conditions for flow acoustics. J. Sound Vib. 332, 714-724.

Golliard, J., Sanna, F., Violato, D. \& AurÉgan, Y. 2016 Measured source term in corrugated pipes with flow. Effect of diameter on pulsation source. AIAA peper 20162886.

INGARD, U. 1959 Influence of fluid motion past a plane boundary on sound reflection, absorption, and transmission. J. Acoust. Soc. Am. 31, 1035-1036.

Khamis, D. \& Brambley, E.J. 2016 Acoustic boundary conditions at an impedance lining in inviscid shear flow. J. Fluid Mech. 796, 386-416.

Khamis, D. \& Brambley, E.J. 2017 Viscous effects on the acoustics and stability of a shear layer over an impedance wall. J. Fluid Mech. 810, 489-534.

Koch, W. \& Mohring, W. 1983 Eigensolutions for liners in uniform mean flow ducts. AIAA J. 21, 200-213. 
Kooijman, G., Hirschberg, A. \& Aurégan, Y. 2010 Influence of mean flow profile and geometrical ratios on scattering of sound at a sudden area expansion in a duct. J. Sound Vib. 329, 607-626.

Kooijman,G., Testud, P., Aurégan, Y. \& Hirschberg, A. 2008 Multimodal method for scattering of sound at a sudden area expansion in a duct with subsonic flow. J. Sound Vib. 310, 902-922.

Marx, D. \& AurÉGan, Y. 2013 Effect of turbulent eddy viscosity on the unstable surface mode above an acoustic liner. J. Sound Vib. 332, 3803-3820.

Marx, D., Aurégan, Y., Bailliet, H., \& Valière, J.-C. 2010 PIV and LDV evidence of hydrodynamic instability over a liner in a duct with flow. J. Sound Vib. 329, 3798-3812.

Myers, M. K. 1980 On the acoustic boundary condition in the presence of flow. J. Sound Vib. 71, 429-434.

Nakiboglu, G., Belfroid, S. P. C., Golliard, J. \& Hirschberg, A. 2011 On the whistling corrugated pipes: Effect of pipe length and flow profile. J. Fluid Mech. 672, 78-108.

Nakiboglu, G. \& Hirschberg, A. 2012 Aeroacoustic power generated by multiple compact axisymmetric cavities: Effect of hydrodynamic interference on the sound production. Phys. Fluids 24, 067101.

Nakiboglu, G., Manders, H. B. M. \& Hirschberg, A. 2012 Aeroacoustic power generated by a compact axisymmetric cavity: prediction of self-sustained oscillation and influence of the depth. J. Fluid Mech. 703, 163-191.

Nennig, B., Renou, Y., Groby, J.-P. \& Aurégan, Y. 2012 A mode matching approach for modeling two dimensional porous grating with infinitely rigid or soft inclusions. J. Acoust. Soc. Am. 131, 3841-3852.

Pascal, L., Piot, E. \& Casalis G. 2017 Global linear stability analysis of flow in a lined duct. J. Sound Vib. 410, 19-34.

Pridmore-Brown, D. C. 1958 Sound propagation in a fluid flowing through an attenuating duct. J. Fluid Mech. 4, 393-406.

Renou, Y. \& AurÉGAn, Y. 2011 Failure of the IngardMyers boundary condition for a lined duct: an experimental investigation. J. Acoust. Soc. Am. 130, 52-60.

Rienstra, S. W. 2003 A classification of duct modes based on surface waves. Wave Motion 37, 119-135.

Rienstra, S. W. \& DARAu, M. 2011 Boundary-layer thickness effects on the hydrodynamic instability along an impedance wall. J. Fluid Mech. 671, 559-573.

Ronneberger, D. AND Jüschke, M. 2007 Sound absorption, sound amplification, and flow control in ducts with compliant walls, In Oscillations, Waves and Interactions (Ed. T. Kurz, U. Parlitz, and U. Kaatze) pp 73-106, Universitätsverlag Göttingen.

Schmid, P.J. \& Henningson, D.S. 2000 Stability and Transition in Shear Flows, Springer.

Schmid, P. J., De PAndo, M. F. \& Peake, N. 2017 Stability analysis for n-periodic array of fluid systems. Phys. Rev. Fluids 2, 113902.

Spillere, A. M. N., Cordioli, J. A. \& BodÉn, H. 2017 On the effect of boundary conditions on impedance eduction results. AIAA peper 2017-3185.

TAM, C. K. W. \& Block, P. J. W. 1978 On the tones and pressure oscillations induced by flow over rectangular cavities. J. Fluid Mech. 89, 373-399.

Tam, C. K.W., Pastouchenko, N. N., Jones, M. G. \& Watson, W. R. 2014 Experimental validation of numerical simulations for an acoustic liner in grazing flow: self-noise and added drag. J. Sound Vib. 333, 2831-2854.

Tester, B.J. 1973 The propagation and attenuation of sound in lined ducts containing uniform or plug flow. J. Sound Vib. 28, 151-203.

Weng, C., Schulz, A., Ronneberger, D., Enghardt, L. \& Bake, F. 2018 Flow and viscous effects on impedance eduction. AIAA J. 56, 1118-1132.

Xin, B., Sun, D., Jing, X. \& Sun, X. 2016 Numerical study of acoustic instability in a partly lined flow duct using the full linearized Navie-Stokes equations. J. Sound Vib. 373, 132146.

Yamouni, S., Sipp, D. \& JacQuin, L. 2013 Interaction between feedback aeroacoustic and acoustic resonance mechanisms in a cavity flow: a global stability analysis. J. Fluid Mech. 717, 134-165. 
ZHANG, Q. \& Bodony, D. J. 2012 Numerical investigation and modelling of acoustically excited flow through a circular orifice backed by a hexagonal cavity. J. Fluid Mech. 693, 367-401.

ZHANG, Q. \& BODONY, D. J. 2016 Numerical investigation of a honeycomb liner grazed by laminar and turbulent boundary layers. J. Fluid Mech. 792, 936-980.

ZiadA, S. \& Shine, S. 1999 Strouhal numbers of flow-excited acoustic resonance of closed side branches. J. Fluid. Struct. 13, 127-142. 